\title{
Appointing high-court judges by political parties
}

\author{
Nicolás Porteiro • Antonio Villar
}

Received: 4 August 2013 / Accepted: 8 August 2014 / Published online: 27 August 2014 (C) The Author(s) 2014. This article is published with open access at SpringerLink.com

\begin{abstract}
This paper proposes a mechanism to overcome the possibility that political parties may block the nomination of High-Court judges when the Parliament is involved in their nomination and their mandate expires on a fixed date. This possibility arises when the default option is that the judge whose mandate expires holds office until an agreement is reached. Our proposal consists of changing the default option by a weighted lottery, related to the shares in Parliament of the different parties. We show that this mechanism is capable of solving the problem under reasonable conditions and implementing a politically balanced solution.
\end{abstract}

Keywords Negotiation - Political competition · Random protocols · Legislative bargaining

\section{JEL Classification D72}

\section{Introduction}

There are a number of relevant instances when political parties have to agree on the choice of candidates for some public institutions. This is the case in many countries for regulatory committees, central bank councils or High Courts. A potential conflict arises when candidates are nominated for a fixed period of office and the norm requires a qualified majority for their nomination. In other words, we may find that the period

Thanks are due to two anonymous referees and the Associate Editor for helpful comments. Financial support from the Spanish Ministry of Economics, under Project ECO2013-43526-R, and Junta de Andalucía, under Project SEJ-6882 (including FEDER funds) is gratefully acknowledged. Unfortunately, Nicolás Porteiro passed away soon after writing this paper.

N. Porteiro · A. Villar $(\varangle)$

Pablo de Olavide University, Seville, Spain

e-mail: avillar@upo.es 
of office of a member of such an institution expires but the parties do not reach an agreement on the person that should replace him/her.

The origin of this paper lies in a real life problem of this sort: the difficulties involved in replacing Spanish Constitutional Court judges over the last few years. This High Court consists of twelve magistrates, eight of whom are chosen in Parliament (four in each of the two Chambers). ${ }^{1}$ The Law establishes that $3 / 5$ favourable votes in Parliament are needed for their nomination. In practice, this requires the consensus of the two largest parties, as neither of them has ever had such a large share of Parliamentary seats, and no other party has enough seats to enable them to reach the necessary share. Each magistrate is appointed for a 9-year period, with a renewal rule that prescribes that one third of the magistrates must be replaced every 3 years. The lack of consensus arose as a major problem in 2007 (the Constitutional Court was established in 1980). Three magistrates stayed in office more than 3 years beyond their duty (and one seat was empty due to the death of another magistrate who was not replaced). Legislators never considered the possibility of such a long delay in the renewal of the Constitutional Court. Indeed, it broke the established renewal pattern, as keeping to the 9-year mandate entailed replacing two thirds of the magistrates on one occasion, one third on next occasion, and none of them the third time. To prevent inconsistency, the law that regulates the Constitutional Court had to be amended, introducing the caveat that the extra period that a magistrate stayed in office was to be discounted from the period of office of his/her substitute. That amendment somehow preserved the renewal norm, even though now some magistrates will stay in office for twice as long as others. Nonetheless, the main issue behind the undue extension of the magistrates' mandate is one of legitimacy. The Spanish Constitutional Court has made very important decisions in such anomalous circumstances, in particular sentences regarding the nature of some Estatutos (the equivalent of the "constitutions" of the Spanish Autonomous Regions). The result is a lack of confidence both in political parties and legislators, the deterioration of the relationships between legislators and the judiciary, and the dissemination of the idea that parties are more concerned about their relative power than about serving citizens. The situation deteriorated so much that some of the magistrates resigned, something unheard of in an institution of this kind.

This problem ${ }^{2}$ derives from the collision of two sensible principles that inspire the norm, or rather from the way in which those principles are implemented. On the one hand, each judge is designated for a fixed term, in order to prevent disproportionate influence of specific individuals on the legal system. On the other, the rule establishes that substituting a judge whose mandate expires requires a qualified majority in Parliament, in order to diminish the effects of the political cycle on the interpretation of the fundamental norms.

The deadlock at which the system can arrive when a judge's mandate expires and Parliament does not have the majority required to approve a substitute, is directly

\footnotetext{
1 The other four magistrates are chosen by the Government and the by the Judges' Council (Consejo General del Poder Judicial).

2 The same problem also affected the Judges' Council (Consejo General del Poder Judicial), where 20 of its 21 members are to be appointed by Parliament. Note that France, Germany and Italy have similar ways of choosing all or some of the judges of their Constitutional Courts.
} 
related to the choice of the status quo as the default option (i.e. the judge whose mandate has expired stays in office until an agreement is reached). Such a default option does not provide the right incentives to reach an agreement. The reason is clear: if the ideology of the judge to be replaced is close to a given party, then this party has no incentive to agree on a different candidate, unless he/she belongs to the same ideological group. When this party is needed for the nomination but cannot ensure a replacement that satisfies its interests, it will block the process.

Here we propose a simple mechanism that is respectful of Parliament and ensures a judge's timely replacement, by simply changing the default option. Once the judge's appointment expires, Parliament is required to make a proposal that satisfies the established quota (3/5 in the case of the Spanish Constitutional Court). If there is agreement, then the candidate is chosen, substitutes the exiting judge, and the process ends. This part is consistent with the current procedure. If there is no agreement, then a weighted lottery applies. Each party presents a candidate who enters a random choice mechanism with a probability weight equal to the proposing party's share in Parliament. The weighted lottery is played and the chosen candidate will be determined by the lottery.

This mechanism is fair because it respects the distribution of power among parties. Firstly, it is consistent with the current system provided that parties are able to reach an agreement in due time. Secondly, when no agreement is reached, on average each party will get its preferred candidate a fraction of times equal to the fraction of its Parliamentary seats.

We show that, under reasonable conditions (single peaked preferences on the ideological traits of the candidates) and a rich variety of candidates over the ideological spectrum, the following holds:

(i) When status quo is the default option, disagreement is the natural outcome;

(ii) When parties are risk averse (or even risk neutral), the weighted lottery mechanism induces agreement between parties before actually recurring to the lottery;

(iii) This two-stage mechanism implements the politically balanced alternative as a Nash equilibrium; and

(iv) The results are robust to the case of bi-dimensional preferences (i.e. when parties care about both the candidates' ideology and their capabilities).

All those results are simple derivations of the non-cooperative theory of bargaining, following the seminal work of Rubinstein (1982), and are closely related to legislative bargaining and arbitration models (Baron and Ferejohn 1989; Banks and Duggan 2006; Cardona and Ponsati 2011; Mylovanov and Zapechelnyuk 2013). Yet they may be useful in suggesting practical ways of solving a relevant problem.

The model aims at highlighting the essential ingredients of the problem and the proposed solution, at the cost of depicting a highly simplified world. In particular: (a) The model focuses on the replacement of one judge at a time; (b) There are only two (relevant) political parties; (c) The utility function of political parties only depends on the ideology of the candidate (a single dimensional variable) and, possibly, on his/her ability (an observable variable that all parties perceive equally). We shall briefly refer to those aspects later on (see Sect. 5).

The rest of the paper goes as follows. The next section sets up the reference model. Section 3 presents the weighted lottery mechanism and characterizes its implications. 
Section 4 briefly elaborates on the consequences of taking not only judges' ideology into account, but also their competence. Finally, Sect. 5 concludes by discussing some other aspects of the problem.

\section{The status quo model}

Let us consider, for the sake of simplicity, that Parliament consists of just two parties, $L$ and $R$. Parliament has to appoint a new High-Court judge by qualified majority $p$, to replace one whose term is over. We assume that neither of the two parties has enough seats in Parliament to reach the qualified majority required, so that they both have to agree on the nomination. $\alpha_{i}$ stands for the share of Parliamentary seats held by party $i=L, R$, with $\alpha_{L}<p$ and $\alpha_{R}<p$, respectively, and $\alpha_{L}+\alpha_{R}=1, p \in(0.5,1]$.

The chosen candidate has to be selected from a pool of candidates in the ideological space $[0,1]$. Assume, for the time being, that all candidates are equally capable of serving as judges (there is no quality dimension in the problem). The current incumbent has ideology $c_{0} \in[0,1]$ and this individual will retain his position until an agreement on her substitute has been reached. Each party has an ideological position defined by a function $V_{i}(c)$ that represents the value it assigns to selecting a judge of ideology $c$. Let $c_{i}$ denote the preferred candidate of party $i=L, R$. That is, $c_{i}$ solves the problem $\max _{c} V_{i}(c)$ subject to $c \in[0,1]$. We assume that preferences are single-peaked, that is, the closer $c$ to $c_{i}$, the greater the utility of party $i$.

Let $c_{L}<c_{R}$, without loss of generality (that is, the closer $c$ is to zero, the more leftist is the chosen candidate).

We say that a candidate is politically balanced when his/her ideology reflects the ideological composition of parliament. In other words, a politically balanced candidate would be one with ideology $c_{\alpha}=\alpha_{L} c_{L}+\alpha_{R} c_{R}$.

Now assume that parties enter into a non-cooperative bargaining process in order to select a candidate to replace an exiting judge. The process can go on for infinite-many periods with the status quo $c_{0}$ prevailing as the default option until a choice is made.

It is easy to show that, within this setting, indefinite disagreement is the most likely outcome, irrespective of the negotiation protocol. Formally:

Proposition 1 If $c_{0} \in\left(c_{L}, c_{R}\right)$ no agreement is reached and the status quo, $c_{0}$, prevails.

Proof To prove this result it is sufficient to consider that, regardless of the details of the negotiation protocol, each party will always play the "safe" strategy of rejecting any offer they receive and making no offer. This strategy ensures that parties obtain $V_{L}\left(c_{0}\right), V_{R}\left(c_{0}\right)$, respectively.

Consider, without loss of generality, a proposal $\hat{c}$ made by party $R$. If party $R$ found it optimal to make the offer it is because $V_{R}\left(c_{0}\right)<V_{R}(\hat{c})$. Moreover, since $c_{0}<c_{R}$, then it necessarily follows that $\hat{c}>c_{0}$. Now, from the perspective of party $L$, if the rejection strategy is not optimal for this offer it is because $V_{L}\left(c_{0}\right)<V_{L}(\hat{c})$ and this, together with $c_{0}>c_{L}$ requires $\hat{c}<c_{0}$, which is a contradiction.

A symmetric argument applies for party $L$ making a proposal. 
This simple result shows that, unless the status quo is too extreme for both parties, negotiations will fail. The reason is that, when the status quo is moderate, any agreement different from $c_{0}$ will imply a loss for one of the parties, preventing any possibility of agreement. Only when the status quo is extreme for both parties there is room for mutual benefit. That is, a necessary (yet not sufficient) condition for agreement is that either $c_{0}<c_{L}$ or $c_{0}>c_{R}$. That situation can only occur if the party that is closest to $c_{0}$ has changed ideology moving towards a more central part of the political spectrum. Otherwise a candidate $c_{0}$ would never have been chosen.

Therefore, when parties' ideologies are stable, non-cooperation is the sure outcome.

\section{Negotiations with deadline and a weighted lottery}

We have seen that negotiations are most likely to fail when the status quo is the default option. Let us define a different choice rule, by changing the default option, which ensures that a new appointment will be obtained in due course, under reasonable assumptions. We first present the rule and then analyse how this new default option affects the possibility of reaching an agreement.

Consider the following two-stage rule:

- Stage 1: Parties open a bilateral negotiation process, in order to reach an agreement with a fixed deadline. If an agreement is obtained, then the chosen candidate is designated and the process ends. If there is no agreement within the established period, we go to stage 2 .

- Stage 2: The two parties are asked to simultaneously name their final candidates, $c_{i}^{f}, i=L, R$. The new judge $c$ is appointed according to a weighted lottery, in such a way that:

$$
c=\left\{\begin{array}{cc}
c_{L}^{f} & \text { with probability } \alpha_{L} \\
c_{R}^{f} & \text { with probability } \alpha_{R}
\end{array}\right.
$$

In order to see how agents behave, consider the final round. Each party will choose $c_{i}^{f}$ in such a way that:

$$
c_{i}^{f} \in \arg \max _{c} \alpha_{i} V_{i}(c)+\left(1-\alpha_{i}\right) V_{i}\left(c_{j}^{f}\right)
$$

with $i=L, R, j=R, L$. It is immediate that Eq. 1 yields $c_{i}^{f}=c_{i}$. That is, each party will propose its preferred candidate as a dominant strategy and there is no scope for manipulating the selection of candidates.

Now the question becomes: is there room for an agreement? We shall see that the answer is positive, provided agents are risk averse.

Let $E V_{i}^{f}=\alpha_{i} V_{i}\left(c_{i}\right)+\left(1-\alpha_{i}\right) V_{i}\left(c_{j}\right)$ be the expected benefit that each party obtains from the final round. And let $\tilde{c}_{i}$ stand for the corresponding certainty equivalent (the point in the ideological spectrum at maximum distance of party $i$ 's ideal point, compatible with reaching an agreement in the first stage). By definition, $\tilde{c}_{L}$ and $\tilde{c}_{R}$ solve: 


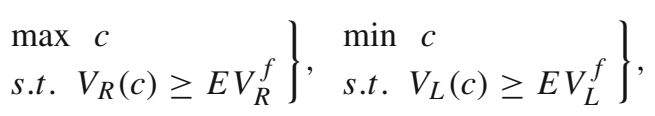

respectively.

Let $c(\pi)=c_{L} \pi+c_{R}(1-\pi)$, with $\pi \in[0,1]$. We say that party $i$ is weakly risk-averse (or has weakly risk-averse preferences) whenever

$$
V_{i}(c(\pi)) \geq \pi V_{i}\left(c_{L}\right)+(1-\pi) V_{i}\left(c_{R}\right), \text { for all } \pi \in[0,1] .
$$

The following result is obtained:

Proposition 2 Assume that both parties have single-peaked and weakly risk-averse preferences. Then, the weighted-lottery mechanism ensures that there is scope for an agreement between the two parties in the first stage. Moreover, the balanced solution $c_{\alpha}$ is always in the set of mutually acceptable candidates.

Proof First, notice that a necessary condition for the weighted-lottery mechanism to induce an agreement in the first stage is that the certainty equivalent of the left party is not below that or the right party, that is, $\tilde{c}_{L} \geq \tilde{c}_{R}$. Otherwise, there is no agreement and the final round is reached.

Let us show now that $\tilde{c}_{L} \geq c_{\alpha} \geq \tilde{c}_{R}$, which trivially implies the condition above. Take first party $L$. From the definition of $\tilde{c}_{L}$ we have that $\tilde{c}_{L} \geq c_{\alpha}$ if and only if,

$$
V_{L}\left(c_{\alpha}\right) \geq E V_{L}^{f} \Leftrightarrow V_{L}\left(\alpha_{L} c_{L}+\alpha_{R} c_{R}\right) \geq \alpha_{L} V_{L}\left(c_{L}\right)+\alpha_{R} V_{L}\left(c_{R}\right)
$$

which is, precisely, the weak risk-aversion condition. The same logic applies for the case of party $R$.

Proposition 2 says that, irrespective of the negotiation protocol, the weighted lottery mechanism induces the parties to agree on an intermediate ideological level, $c^{\prime} \in$ $\left[\tilde{c}_{L}, \tilde{c}_{R}\right]$, without actually recurring to the lottery. Note that this result does not require any particular functional form and holds for any single peaked and risk-averse or risk neutral utility function.

The second part of Proposition 2 can be rephrased as follows:

Corollary 1 The choice of the balanced candidate can be implemented as a Nash equilibrium by the weighted-lottery mechanism.

\section{The choice problem when both ideology and ability matter}

Consider now the more general case in which the parties' preferences are bidimensional: they care both about the ideology $(c)$ and the ability $(a)$ of the candidates. Utilities, therefore, take the form $V_{i}(c, a)$, for $i \in\{L, R\}$. We assume, as before, that utilities are single peaked with respect to the ideological component. That is, $V_{i}(c, a)$ is increasing (resp. decreasing) in $c$ provided $c<c_{i}$ (resp. $c>c_{i}$ ). 
Concerning the ability dimension we assume that utilities are increasing in $a$ for both parties, and that they agree on the measurement of ability, that varies in a compact interval $\left[a_{\min }, a^{\max }\right] .^{3}$

The following result is quite straightforward,

Proposition 3 Assume that $V_{i}(c, a)$ is single-peaked and risk-averse with respect to $c$ and increasing in $a$, for $i=L, R$. Then, the weighted-lottery mechanism induces an agreement on some candidate $c^{\prime}$ such that, there is no other candidate with ideology $c^{\prime}$ and a higher ability.

Proof Let $a_{c}^{\max }$ denote the highest ability attainable for a candidate with ideology $c$. As utilities are increasing in $a$ for both parties, then a change from any $a \neq a_{c}^{\max }$ to $a_{c}^{\max }$ would imply an increase in the value obtained by both parties. Therefore, $a \neq a_{c}^{\max }$ can never be an equilibrium.

Define now $\tilde{V}_{i}(c)=V_{i}\left(c, a_{c}^{\max }\right)$ for each $c \in[0,1]$. Then Proposition 2 brings the desired result.

An example of a situation in which the weighted lottery mechanism works well is when there is always a high-competent candidate for every ideological level. Formally, this corresponds to a situation where, for every $c$ it holds that $a_{c}^{\max }=a^{\max }$.

Note that the balanced candidate, $\left(c_{\alpha} ; a_{c_{\alpha}}^{\max }\right)$, can also be implemented as a Nash equilibrium.

\section{Discussion}

We have presented a very simple model that suggests a way of solving the timely renewal of magistrates in some High Courts. We have shown that when political parties have to agree on the replacement of a High-Court judge, designated for a fixed term, the default option significantly affects the incentives to reach an agreement. When holding office until the agreement is reached is the default, no agreement should be expected. When the default is a weighted lottery, then agreement will most likely occur, prior to the lottery. ${ }^{4}$

The model's extreme simplicity enables us to focus on the key aspects of the problem and reach clear-cut conclusions. By the same token, a number of relevant aspects that affect the appointment process in real life have been put aside. They include the following.

The role of discounting. In our two-stage model no time discount has been contemplated, because we implicitly assume that the period to reach an agreement is

\footnotetext{
3 This implies that party $L$ would prefer a more able candidate, even if it is the one preferred by party $R$, and viceversa. One may well think that in real life there are strategic aspects of a higher level according to which a bad candidate of the other party may be regarded as convenient (either to discredit the proposing party or to be less influential in the Court). We shall not consider here this aspect.

${ }^{4}$ Note that the lottery plays here the role of final offer arbitration. Mylovanov and Zapechelnyuk (2013) show that, in NTU environments, this type of arbitration is superior to conventional arbitration. The key reason is that "The divergence of proposals and the restriction that the arbitrator must pick a side and cannot modify the proposals ... provides incentives for the parties to agree on a socially optimal outcome prior to the arbitration." The same basic argument applies here.
} 
short. When the period enabled to reach an agreement is long enough and the status quo prevails in the meantime, results may change. More specifically, impatience may affect the parties' strategies and there will be a threshold beyond which it could be preferable waiting until the lottery applies. ${ }^{5}$

The existence of a finite number of candidates. We have assumed that there are enough eligible judges to fully cover the political spectrum. When this is not the case, the results may also change. Things would not change much provided there is enough variety spreading evenly over the political spectrum. Otherwise results will depend on the distribution of the candidates. Be as it may, the case of discrete alternatives always makes more difficult the model and entails multiple equilibria. ${ }^{6}$

Only two parties involved. The model assumes that there are only two parties so that the universe of outcomes is either unanimity or disagreement. This is a reasonable assumption in order to describe situations in which there are two majority parties whose agreement is needed to make an appointment. The many-party case involves a more complex model where the relative ideological proximity and the degree of risk aversion will play a role in the solution of the game. The resulting equilibria will typically depend on the ranges of utility parameters and ideological positions.

Log-rolling options. Log-rolling (Buchanan and Tullock 1962) refers here to the possibility of making agreements in a higher dimensional space of strategies in which compensations can take place. This larger space may involve time (here the repeated nature of the game becomes relevant), issues (considering other committees in which similar agreements are required), or quotas (apportioning candidates when more than one is to be appointed). ${ }^{7}$

More complex utility functions. The model has depicted political parties whose utilities only depend on the ideology of the candidate and on her ability (singledimensional and observable variables). Actual political parties have certainly richer utility functions, which include aspects such as "social responsibility", concern for the public opinion, the existence of a reference point (e.g, status quo dependent utilities), or the presence of externalities (e.g. making the decision depending on the ideological composition of the resulting group).

Incomplete information. All relevant ingredients of the choice problem are assumed to be publicly known. Each decision maker observes the ideology profile and quality of the candidates and knows the utility function of the other party. Real life decision usually involve incomplete or asymmetric information, which affects the resulting equilibria.

\footnotetext{
5 See Porteiro (2007) for an analysis of a negotiation protocol that explicitly accounts for discounting in the presence of a status quo and where the level of patience has direct implications on the equilibrium negotiation strategies of players.

6 From a technical perspective, moving from a bargaining problem over a perfectly divisible issue to one where there are indivisibilities, is far from straightforward. As the paper by Damme et al. (1990) shows, introducing indivisibilities in the alternating-offer bargaining game of Rubinstein (1982) eliminates the uniqueness of equilibrium (there are infinite subgame perfect equilibria, including very inefficient ones).

7 When there are several candidates that can be simultaneously replaced, the parties' strategies become more complex, depending on the ideological composition of the exiting judges and the changes in the parties' shares of seats in Parliament during the reference period. One can find different solutions in the first stage depending on those aspects, so that agreement may be the outcome under some circumstances.
} 
Simplicity helps understanding the basic features of the problem and some lines of solution, as illustrated by our model. Yet one has to bear in mind that in a richer scenario we shall find agreements in many cases, even if the status quo is the default option. Moreover, in a more realistic setting, there will cases in which the weighted lottery of the proposed mechanism will actually be played. This does not invalidate our proposal, as deadlock still arises in even those richer environments. On the contrary, since the lottery is a way of avoiding deadlocks that is respectful with the distribution of power in Parliament, this new default option is superior to the existing one as it ensures replacement in due time.

Finally, one may argue that our proposal is a totally artificial mechanism that goes against the culture of lawyers and politicians. This is not the case. A lottery mechanism, known as insaculation, was used for almost four centuries in Spain to appoint public officers at different levels. This choice procedure was introduced in the fourteenth century in order to avoid the excessive influence of some families in the management of public affairs. A set of candidates was first selected and then pieces of paper with their names were introduced in a bag (sacculum, in Latin) from which one was chosen at random. Our proposal here may be regarded as following this tradition. ${ }^{8}$

\section{References}

Banks JS, Duggan J (2006) A general bargaining model of legislative policy-making. Q J Polit Sci 1:49-85 Baron D, Ferejohn J (1989) Bargaining in legislatures. Am Polit Sci Rev 83:1181-1206

Buchanan JM, Tullock G (1962) The calculus of consent: logical foundations of constitutional democracy. University of Michigan Press, Ann Arbor

Cardona D, Ponsati C (2011) Uniqueness of stationary equilibria in bargaining one dimensional policies under (super) majority rules. Games Econ Behav 73:65-75

Mylovanov T, Zapechelnyuk A (2013) Optimal arbitration. Inter Econ Rev 54:769-785

Porteiro N (2007) An efficient and egalitarian negotiation procedure for economies with externalities. Soc Choice Welfare 28:19-40

Rubinstein A (1982) Perfect equilibrium in a bargaining model. Econometrica 50:97-109

van Damme E, Selten R, Winter E (1990) Alternating bid bargaining game with a smallest money unit. Games Econ Behav 2:188-201

\footnotetext{
8 Let us recall here that, faced with a similar deadlock problem, the Catholic Church decreed in 1274 that those cardinals that should elect a new Pope be locked in seclusion cum clave ("with a key") and not permitted to leave until an agreement had been reached. That is still another way of implementing the choice procedure in a limited time span.
} 